\title{
Tratamento cirúrgico da escoliose em pacientes com amiotrofia espinhal com parafusos pediculares (instrumental de $3^{\mathfrak{a}}$ geração) e complicações precoces
}

Surgical treatment of scoliosis in spinal muscular atrophy with pedicle screws (third generation instrumentation) and early complications Tratamiento quirúrgico de la escoliosis en pacientes con amiotrofia espinal con tornillos pediculares (instrumental de $3^{\underline{a}}$ generación) y complicaciones precoces

\author{
Daniel Cantarelli dos Santos' \\ Paulo Tadeu Maia Cavali² \\ Marcus Alexandre Mello Santos ${ }^{3}$ \\ Maurício Antonelli Lehoczki ${ }^{3}$ \\ Alexander Junqueira Rossato ${ }^{3}$ \\ Elcio Landim ${ }^{4}$
}

\section{RESUMO}

Objetivo: avaliar a utilização de artrodese via posterior com parafusos pediculares para correção da escoliose em pacientes com amiotrofia espinhal. Métodos: realizou-se um estudo retrospectivo de 16 pacientes com amiotrofia espinhal submetidos à artrodese via posterior exclusiva, com parafusos pediculares. Foi avaliado o perfil geral dos pacientes e o potencial de correção do ângulo de Cobb e da obliquidade pélvica, além das complicações precoces. Resultados: o ângulo de Cobb pré-operatório foi em média de $94,6^{\circ}\left(65\right.$ a $\left.132^{\circ}\right)$, no pós-operatório de $40,4^{\circ}\left(2\right.$ a $\left.70^{\circ}\right)$, percentual de correção de $57,2 \%$. A obliquidade pélvica pré-operatória foi em média $34,7^{\circ}$ $\left(25\right.$ a $\left.56^{\circ}\right)$, no pós-operatório foi para

\section{ABSTRACT}

Objectives: to report the results on the treatment of scoliosis in spinal muscular atrophy, using posterior arthrodesis with pedicle screws. Methods: a retrospective study was carried out with 16 patients who underwent posterior spinal fusion with pedicle screws. The general status of the patients, correction of the Cobb angle, correction of pelvic obliquity and early complications were analyzed. Results: the initial Cobb angle mean was 94.6 (65 to $132^{\circ}$ ) turning into $40,4^{\circ}\left(2\right.$ to $20^{\circ}$ ) after the surgery, correction of $57.2 \%$. The initial pelvic obliquity mean was $34.7^{\circ}\left(25\right.$ to $\left.56^{\circ}\right)$ turning into $11.3^{\circ}\left(0\right.$ to $\left.20^{\circ}\right)$, correction of 67.4\%. Conclusions: the treatment

\section{RESUMEN}

Objetivo: evaluar la utilización de la artrodesis vía posterior con tornillos pediculares para la corrección de la escoliosis en pacientes con amiotrofia espinal. Métodos: fue realizado un estudio retrospectivo de 16 pacientes con amiotrofia espinal sometidos a la artrodesis vía posterior exclusiva, con tornillos pediculares. Fue evaluado el perfil general de los pacientes, y el potencial de corrección del ángulo de Cobb y de la oblicuidad pélvica, además de las complicaciones precoces. Resultados: el ángulo de Cobb preoperatorio fue en promedio $94.6^{\circ}\left(65\right.$ a $\left.132^{\circ}\right)$, en el postoperatorio $40.4^{\circ}$ (2 a 70), porcentual de corrección de $57.2 \%$. La oblicuidad pélvica preoperatoria fue en media $34.7^{\circ}\left(25\right.$ a $\left.56^{\circ}\right)$, en el posto-

\footnotetext{
Trabalho realizado na Associação de Assistência à Criança Deficiente - AACD - São Paulo (SP), Brasil.

'Estagiário em Cirurgia de Coluna da Assistência à Criança Deficiente - AACD - São Paulo (SP), Brasil; e do Grupo de Coluna do Hospital das Clínicas da Faculdade de Ciências Médicas da Universidade Estadual de Campinas - UNICAMP - Campinas (SP), Brasil.

${ }^{2}$ Mestre; Médico Ortopedista do Grupo de Escoliose da Associação de Assistência à Criança Deficiente - AACD - São Paulo (SP), Brasil; e do Grupo de Coluna do Hospital das Clínicas da Faculdade de Ciências Médicas da Universidade Estadual de Campinas - UNICAMP - Campinas (SP), Brasil.

${ }^{3}$ Assistente do Grupo de Escoliose da Associação de Assistência à Criança Deficiente - AACD - São Paulo (SP), Brasil.

${ }^{4}$ Doutor; Chefe do Grupo de Escoliose da Associação de Assistência à Criança Deficiente - AACD - São Paulo (SP), Brasil; Professor Adjunto e Chefe do Grupo de Cirurgia da Coluna Vertebral do Departamento de Ortopedia e Traumatologia do Hospital das Clínicas da Faculdade de Ciências Médicas da Universidade Estadual de Campinas - UNICAMP - Campinas (SP), Brasil.
} 
$11,3^{\circ}\left(0\right.$ a $\left.20^{\circ}\right)$, com percentual de correção de $67,4 \%$. Cinco pacientes tiveram complicações precoces $(31,2 \%)$ com boa resolução. Conclusões: o tratamento cirúrgico da escoliose em pacientes com amiotrofia espinhal por meio de artrodese via posterior utilizando parafusos pediculares tem grande potencial de correção da deformidade coronal e da obliquidade pélvica, sem grandes complicações no pós-operatório precoce.

DESCRITORES: Atrofia muscular espinhal; Escoliose/cirurgia; Artrodese; Fusão vertebral; Instrumentos cirúrgicos

of scoliosis in spinal muscular atrophy using posterior arthrodesis with pedicle screws presents a great potential of correction for the coronal deformity and pelvic obliquity, without serious early complications.

KEYWORDS: Muscular atrophy, spinal; Scoliosis/surgery; Arthrodesis; Spinal fusion; Surgical instruments peratorio fue para $11.3^{\circ}\left(0\right.$ a $\left.20^{\circ}\right)$, porcentual de corrección de 67.4\%. Cinco pacientes tuvieron complicaciones precoces (31.2\%) con buena resolución. Conclusión: el tratamiento quirúrgico de la escoliosis en pacientes con amiotrofia espinal, con artrodesis vía posterior utilizando tornillos pediculares, tiene gran potencial de corrección de la deformidad coronal y de la oblicuidad pélvica, sin grandes complicaciones en el postoperatorio precoz.

DESCRIPTORES: Atrofia muscular espinal; Escoliosis/cirugía; Artrodesis; Fusión espinal; Instrumentos quirúrgicos

\section{INTRODUÇÃO}

Amiotrofia espinhal (AME) inclui um grupo de patologias neuromusculares autossômicas recessivas que envolve os neurônios motores da porção ventral da medula e núcleo motor cerebral ${ }^{1,2}$.

É a mais comum doença neuromuscular fatal da infância, e o terceiro diagnóstico neuromuscular mais frequente até os 18 anos de idade, com prevalência de oito para cada 100 mil nascidos vivos ${ }^{3}$. Os pacientes apresentam fraqueza, hipotonia, arreflexia e tremores com sensibilidade mantida e inteligência normal. A fraqueza muscular tem início nos membros inferiores e nos músculos proximais ao tronco mais do que nos distais ${ }^{4}$.

A classificação da AME é dividida em três tipos e se correlaciona com a gravidade da doença e a idade do aparecimento ${ }^{5}$.

A prevalência da escoliose é proporcional à condição de marcha do paciente e à gravidade da doença. Compromete cerca de $100 \%$ dos pacientes do tipo 1 e 2 , e a totalidade dos pacientes do tipo 3. As curvas típicas do padrão neuromuscular apresentam forma de "C" longo, com curva única em $90 \%$ dos casos e mais frequente à direita. Destas, $80 \%$ são toracolombares e $20 \%$ torácicas. A flexibilidade é maior do que nas curvas idiopáticas, porém com uma velocidade de progressão aumentada e associada à cifose $\mathrm{e}^{3}$.

A história natural inclui progressão grave da curva, com impotência funcional inclusive dos membros superiores associada ao frágil suporte do tronco. Além disso, os pacientes com AME têm falência respiratória progressiva pela fraqueza da musculatura intercostal e diafragmática, agravada pela escoliose que necessita de estabilização $0^{3-7}$.

O objetivo deste trabalho foi descrever o uso do material de terceira geração na correção da escoliose em pacientes com amiotrofia espinhal e seu potencial de correção.

\section{MÉTODOS}

Foi realizada uma revisão retrospectiva dos pacientes com amiotrofia espinhal submetidos ao tratamento cirúrgico de escoliose via posterior com uso de parafusos pediculares na Associação de Assistência à Criança Deficiente de São Paulo (AACD-SP). No levantamento de 2004 até 2009, havia registro de 29 pacientes operados, sendo excluídos do estudo 13 pacientes por falta de radiografias ou registros detalhados no prontuário, totalizando 16 pacientes no estudo.

Ao todo, 8 pacientes eram do sexo masculino (50\%) e 8 do sexo feminino (50\%). A idade média na data da cirurgia era de 14,8 anos (variação de 9 a 23 anos) e o peso médio era 47,5 kg (variação de 25 a $61 \mathrm{~kg}$ ). Quanto ao tipo de amiotrofia, 14 eram do tipo $2(87,5 \%)$, e 2 eram do tipo $3(12,5 \%)$. Não foi operado nenhum paciente com diagnóstico de amiotrofia espinhal tipo 1 no estudo (Tabela 1).

Dos 16 pacientes operados, 13 apresentaram curva toracolombar para a esquerda $(81,3 \%), 2$ tinham curva toracolombar para a direita $(12,5 \%)$ e um paciente apresentava curva lombar à esquerda $(6,3 \%)$. O nível de artrodese variou quanto à vértebra proximal, já que todas se estenderam até o ilíaco. Foram 7 casos da quinta vértebra torácica (T5) ao ilíaco (43,8\%), 4 casos de T3 ao ilíaco (25\%), 3 casos de T2 ao ilíaco (18,8\%), e 2 casos de T5 ao ilíaco $(12,5 \%)$. Foram instrumentados 19,9 pedículos em média (variação de 16 a 23) por cirurgia.

Estes pacientes foram encaminhados, já com o diagnóstico de amiotrofia espinhal, do ambulatório de doenças neuromusculares da instituição, sendo referenciados para o grupo de escoliose que define se o caso é elegível ou não para o procedimento cirúrgico. A indicação cirúrgica ocorre nas escolioses de alto grau (superior a $45^{\circ}$ ), progressivas e com obliquidade pélvica acentuada (superior a $\left.20^{\circ}\right)^{4,6}$. Realiza-se avaliação clínica pré-operatória 
TABELA 1 - Perfil dos pacientes operados, nível de artrodese, número de pedículos instrumentados e complicações precoces

\begin{tabular}{|c|c|c|c|c|c|c|c|c|c|c|}
\hline Nome & $\begin{array}{l}\text { Sexo } \\
(M / F)\end{array}$ & Idade* & $\begin{array}{c}\text { Peso } \\
(\mathrm{kg})\end{array}$ & Diagnóstico & Cobb & $\begin{array}{l}\text { OP } \\
\text { pré }\end{array}$ & $\begin{array}{c}\text { Tipo de } \\
\text { curva }\end{array}$ & $\begin{array}{c}\text { Nível da } \\
\text { artrodese }\end{array}$ & $\mathrm{nPI}$ & Complicações \\
\hline CCAS & $\mathrm{F}$ & 12 & 48 & AME-II & 115 & 32 & TLE & T2-I & 19 & Infecção \\
\hline JMSSA & $\mathrm{F}$ & 10 & 39 & AME-II & 88 & 36 & TLE & T4-I & 20 & \\
\hline NLB & $\mathrm{F}$ & 12 & 44 & AME-II & 72 & 25 & TLE & T2-I & 18 & \\
\hline ASR & $\mathrm{F}$ & 18 & 48 & AME-II & 132 & 56 & TLE & T4-I & 19 & \\
\hline LMS & $\mathrm{F}$ & 23 & 48 & AME-III & 65 & 43 & TLE & T5-I & 22 & Deiscência \\
\hline MMP & M & 19 & 60 & AME-II & 65 & 32 & LE & T4-I & 20 & \\
\hline SCC & M & 14 & 52 & AME-II & 70 & 26 & TLE & T3-I & 20 & \\
\hline DCF & M & 16 & 50 & AME-II & 92 & 33 & TLE & T3-I & 22 & \\
\hline CWAO & M & 19 & 60 & AME-II & 100 & 30 & TLE & T2-I & 22 & \\
\hline CRO & $\mathrm{F}$ & 12 & 41 & AME-II & 115 & 25 & TLD & T5-I & 16 & Infecção \\
\hline $\mathrm{CHCS}$ & M & 18 & 59 & AME-II & 90 & 30 & TLE & T4-I & 20 & \\
\hline DSF & $\mathrm{F}$ & 11 & 50 & AME-II & 98 & 30 & TLE & T4-I & 20 & \\
\hline GMO & M & 9 & 35 & AME-II & 108 & 34 & TLE & T3-I & 20 & \\
\hline WVAS & M & 12 & 25 & AME-II & 100 & 44 & TLD & T3-I & 18 & Parestesia \\
\hline RPF & $F$ & 17 & 40 & AME-II & 108 & 39 & TLE & T4-I & 23 & \\
\hline $\mathrm{HPL}$ & $M$ & 15 & 61 & AME-III & 96 & 41 & TLE & T4-I & 20 & Deiscência \\
\hline
\end{tabular}

*Idade em anos na data da cirurgia.

OP: obliquidade pélvica; TLD: curva toracolombar direita; TLE: curva toracolombar esquerda; LE: curva lombar esquerda; nPI: número de pedículos instrumentados.

multidisciplinar. O pneumologista avalia a capacidade pulmonar e define a liberação para o procedimento cirúrgico (Figuras 1 e 2).

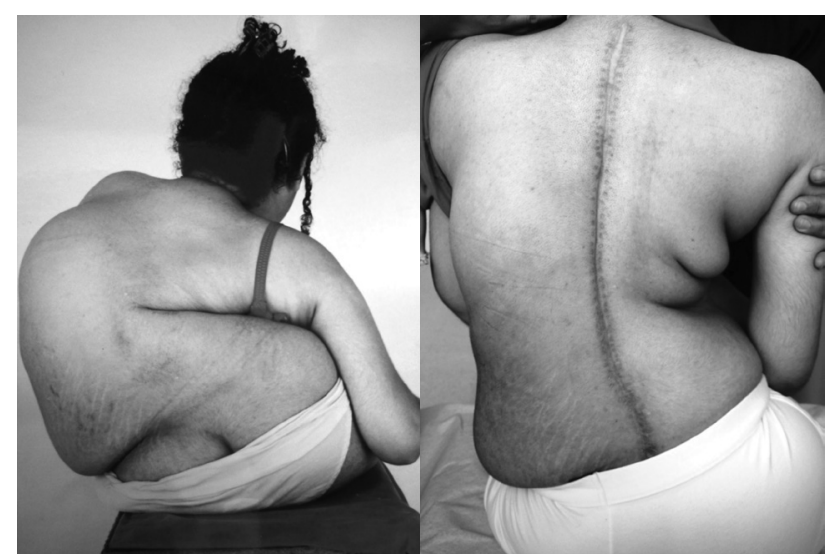

Figuras 1 e 2

Aspecto clínico da paciente ASR com AME tipo 2 pré e pósoperatório.

Foi avaliado no estudo pré-operatório o tipo de curva, com base na vértebra do ápice da convexidade, valor do ângulo de Cobb no plano coronal e valor da obliquidade pélvica. Estes dados apresentam boa confiabilidade como parâmetros radiográficos na escoliose neuromuscular ${ }^{8}$, aferidos pelos métodos recomendados pelo Spinal Deformity Group ${ }^{9}$. No pós-operatório, foi avaliado nível de artrodese, quantidade de pedículos instrumentados (com parafusos e alguns ganchos), ângulo de Cobb e obliquidade pélvica pós-correção, os respectivos percentuais de correção (Tabelas 2 e 3), além das complicações cirúrgicas pós-operatórias precoces (até três meses do pós-operatório) ${ }^{10}$.

\section{TABELA 2 - Correção do ângulo de Cobb}

\begin{tabular}{lcccc}
\hline Nome & Cobb pré & Cobb pós & $\begin{array}{c}\text { Correção } \\
\text { (graus) }\end{array}$ & $\begin{array}{c}\% \text { de } \\
\text { correção }\end{array}$ \\
\hline CCAS & 115 & 20 & 95 & 82 \\
JMSSA & 88 & 30 & 58 & 65 \\
NLB & 72 & 28 & 44 & 61 \\
ASR & 132 & 60 & 72 & 5 \\
LMS & 65 & 50 & 15 & 23 \\
MMP & 65 & 2 & 63 & 87 \\
SCC & 70 & 4 & 66 & 94 \\
DCF & 92 & 42 & 50 & 54 \\
CWAO & 100 & 50 & 50 & 50 \\
CRO & 115 & 50 & 65 & 56 \\
CHCS & 90 & 40 & 50 & 55 \\
DSF & 98 & 42 & 56 & 57 \\
GMO & 108 & 48 & 60 & 55 \\
WVAS & 100 & 70 & 30 & 30 \\
RPF & 108 & 66 & 42 & 38 \\
HPL & 96 & 45 & 51 & 53 \\
\hline
\end{tabular}

\section{Técnica cirúrgica}

Para todos os pacientes, foi realizada correção seguida de artrodese da curva por via posterior em um único tempo cirúrgico. O paciente, após anestesia geral, foi colocado em decúbito ventral com coxins de silicone em tórax, quadril e face. Realizada exposição da coluna por 
TABELA 3 - Correção da obliquidade pélvica

\begin{tabular}{|c|c|c|c|c|}
\hline Nome & OP pré & OP pós & $\begin{array}{c}\text { Correção } \\
\text { (graus) }\end{array}$ & $\begin{array}{c}\% \text { de } \\
\text { correção }\end{array}$ \\
\hline CCAS & 32 & 10 & 20 & 68 \\
\hline JMSSA & 36 & 0 & 36 & 100 \\
\hline NLB & 25 & 6 & 19 & 76 \\
\hline ASR & 56 & 17 & 39 & 69 \\
\hline LMS & 43 & 12 & 31 & 72 \\
\hline MMP & 32 & 8 & 24 & 75 \\
\hline SCC & 26 & 0 & 26 & 100 \\
\hline DCF & 33 & 18 & 15 & 45 \\
\hline CWAO & 30 & 4 & 26 & 86 \\
\hline $\mathrm{CRO}$ & 25 & 15 & 10 & 40 \\
\hline $\mathrm{CHCS}$ & 30 & 15 & 15 & 50 \\
\hline DSF & 30 & 11 & 19 & 63 \\
\hline GMO & 34 & 17 & 17 & 50 \\
\hline WVAS & 44 & 20 & 24 & 54 \\
\hline RPF & 39 & 10 & 29 & 74 \\
\hline $\mathrm{HPL}$ & 41 & 18 & 23 & 56 \\
\hline
\end{tabular}

OP: obliquidade pélvica.

incisão mediana e dissecção subperiostal com auxílio de bisturi elétrico até os dois ilíacos. Para instrumentação com parafusos pediculares, foi utilizada a "técnica de mãos livres"11 e o controle fluoroscópico final. A curva foi corrigida com a montagem das duas hastes utilizando os princípios de translação, derrotação, compressão e distração conforme necessário, respeitando o limite do implante e resistência óssea do paciente. Foi realizada decorticação das facetas e lâmina. Foram retirados os processos espinhosos que, depois de triturados, são utilizados na enxertia do leito de artrodese já preparado. Realizou-se fechamento por planos com dreno de aspiração. É sempre realizada profilaxia com antibióticos e o pós-operatório imediato acontece em unidade de terapia intensiva (Figuras 3 e 4 ).

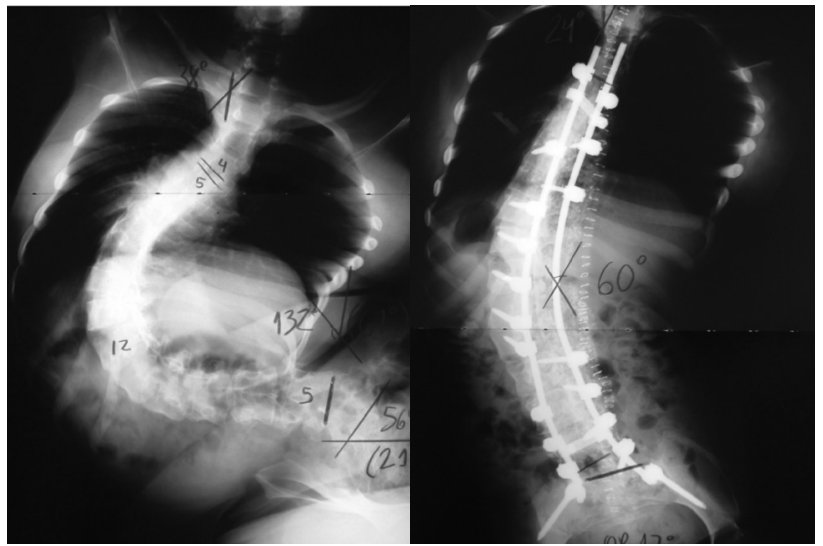

Figuras 3 e 4

Aspecto radiológico da paciente $A S R$ pré $\left(\right.$ Cobb $=132^{\circ} \mathrm{e}$ $\left.O P=56^{\circ}\right)$ e pós-operatório $\left(\mathrm{Cobb}=60^{\circ}\right.$ e $\left.\mathrm{OP}=17^{\circ}\right)$.

\section{Análise estatística}

Com os dados obtidos, foi realizada uma análise descritiva, calculando-se frequências e porcentagens para as variáveis qualitativas (Tabela 4) e medidas descritivas para variáveis quantitativas (Tabela 5). Para testar as variáveis medidas pré e pós-tratamento, foi utilizado o teste de Wilcoxon pareado.

TABELA 4 - Frequências e porcentagens para variáveis: sexo, diagnóstico, nível, complicações e tipo de curva

\begin{tabular}{|c|c|c|}
\hline Variável & Frequência & $\%$ \\
\hline \multicolumn{3}{|l|}{ Sexo } \\
\hline Feminino & 8 & 50 \\
\hline Masculino & 8 & 50 \\
\hline Total & 16 & 100 \\
\hline \multicolumn{3}{|l|}{ Diagnóstico } \\
\hline AME-II & 14 & 87,5 \\
\hline AME-III & 2 & 12,5 \\
\hline Total & 16 & 100 \\
\hline \multicolumn{3}{|l|}{ Nível } \\
\hline T2-I & 3 & 18,8 \\
\hline T3-I & 4 & 25,0 \\
\hline T4-I & 7 & 43,8 \\
\hline T5-I & 2 & 12,5 \\
\hline Total & 16 & 100,0 \\
\hline \multicolumn{3}{|l|}{ Complicações } \\
\hline Deiscência FO & 2 & 12,5 \\
\hline Infecção FO & 2 & 12,5 \\
\hline Parestesia sentado & 1 & 6,2 \\
\hline Total & 5 & 31,2 \\
\hline \multicolumn{3}{|l|}{ Tipo de curva } \\
\hline Lombar E & 1 & 6,3 \\
\hline Toracolombar D & 2 & 12,5 \\
\hline Toracolombar E & 13 & 81,3 \\
\hline Total & 16 & 100 \\
\hline
\end{tabular}

FO: ferida operatória.

TABELA 5 - Estatísticas descritivas para as variáveis quantitativas

\begin{tabular}{lccccc}
\hline Variáveis & $\mathbf{n}$ & Mínimo & Máximo & Média & $\begin{array}{c}\text { Desvio } \\
\text { padrão }\end{array}$ \\
\hline Idade & 16 & 9 & 23 & 14,81 & 3,94 \\
Peso $(\mathrm{kg})$ & 16 & 25 & 61 & 47,50 & 10,02 \\
Cobb pré & 16 & 65 & 132 & 94,62 & 19,32 \\
OP pré & 16 & 25 & 56 & 34,75 & 8,23 \\
nPI & 16 & 16 & 23 & 19,94 & 1,74 \\
Cobb pós & 16 & 2 & 70 & 40,44 & 19,59 \\
$\%$ Cobb & 16 & 23 & 94 & 57,13 & 18,82 \\
OP pós & 16 & 0 & 20 & 11,31 & 6,37 \\
$\%$ OP & 16 & 40 & 100 & 67,44 & 18,02 \\
\hline OP: obliquidade pélvica; nPI: número de pedículos instrumentados.
\end{tabular}


Em todos os testes, o nível de significância adotado foi de $5 \%$ ou o valor de $\mathrm{p}$ correspondente. Todas as análises foram feitas no programa SPSS for Windows, v.12.

\section{RESULTADOS}

$\mathrm{O}$ ângulo de Cobb coronal médio das curvas operadas foi de $94,6^{\circ}$ (variação de 65 a $132^{\circ}$ ), e após a cirurgia o Cobb médio foi de $40,4^{\circ}$ (variação de dois a $70^{\circ}$ ), uma correção média de $54,2^{\circ}\left(57,2^{2} \%\right)$. Para obliquidade pélvica pré-operatória, o valor médio foi de $34,7^{\circ}$ (variação de 25 a $56^{\circ}$ ), e no pós-operatório a obliquidade pélvica média foi de $11,3^{\circ}$ (variação de 0 a $20^{\circ}$ ), com uma correção média de $23,4^{\circ}(67,4 \%)$.

Cinco pacientes apresentaram complicações pós-operatórias precoces $(31,2 \%)$. Dois casos de infecção $(12,5 \%)$, sendo uma superficial com resolução após curativos e antibioticoterapia endovenosa, uma infecção profunda que necessitou de limpeza cirúrgica e antibioticoterapia endovenosa, com boa resolução sem necessidade de retirada do implante. Ocorreu deiscência na ferida pós-operatória em dois pacientes (12,5\%), ambas resolvidas com desbridamento das bordas e nova sutura, sem necessidade de retalho ou intervenção da cirurgia plástica. Um paciente apresentou parestesia $(6,2 \%)$ em um membro inferior ao se manter sentado na cadeira durante o pós-operatório que foi resolvida após adaptação da cadeira de rodas. Este paciente foi o que manteve a maior obliquidade pélvica residual $\left(20^{\circ}\right)$, o que poderia justificar o quadro clínico.

Pelo teste de Wilcoxon pareado para ângulo de Cobb e obliquidade pélvica, notou-se diferença significativa nos valores obtidos nos períodos pré e pós-operatório.

\section{DISCUSSÃO}

Ainda não existe tratamento medicamentoso específico para AME, mas há um aumento da expectativa de vida dos pacientes que se deve ao melhor manejo pulmonar, com técnicas de ventilação intermitente sob pressão positiva, e importante correção da escoliose $e^{3,7}$.

Utilizamos o método proposto por Byers e Banker ${ }^{5}$ na classificação dos pacientes com AME, dividida em três tipos:

- AME tipo 1 ou forma aguda da doença de WerdnigHoffman. Tipo mais severo de AME, diagnosticado até os seis meses, com alta incidência de falência respiratória e óbito antes dos dois anos de idade.

- AME tipo 2 ou forma crônica da doença de WerdnigHoffman. Tipo intermediário da doença com início dos sintomas dos 6 aos 18 meses de idade. As crianças são muito fracas, com dificuldade para andar sem auxílio e alguns sentam sem apoio. A sobrevida é variável, com pacientes sobrevivendo até a terceira ou quarta década. A fraqueza muscular afeta, de início, os membros inferiores e os músculos proximais ao tronco mais que os distais.

- AME tipo 3 ou doença de Kugelberg-Welander. Forma que se apresenta após os 18 meses de idade. Muitas vezes, a criança tem uma marcha, mesmo com fraqueza da musculatura proximal do quadril, podendo deambular até a terceira década e com leve comprometimento dos membros superiores ${ }^{6}$.

A idade de início da escoliose varia com a gravidade da doença, o tipo 1 ocorre antes dos 2 anos, entre 1 e 7 anos no tipo 2 e entre 4 e 14 anos no tipo $3^{3}$. Em nosso estudo, foram operados apenas pacientes com AME tipo 2 e 3 , que são os que se beneficiam da cirurgia na $2^{\mathrm{a}}$ década de vida.

$\mathrm{O}$ manejo desses pacientes com órteses pode ser utilizado para diminuir a progressão da escoliose e tentar postergar a cirurgia nos pacientes mais jovens, entretanto não é um tratamento definitivo, pois essas curvas são progressi$\operatorname{vas}^{3,4,6}$. Além disso, o uso do colete tende a agravar a pneumopatia restritiva, sendo descontinuado pelos pacientes ${ }^{8}$.

Embora a recomendação para cirurgia sejam curvas progressivas acima de $50^{03,4,6}$, a maioria das séries aborda pacientes com curvas coronais maiores ${ }^{12-14}$, demonstrando a dificuldade de prever o ritmo de progressão e aumento da rigidez dessas curvas. O objetivo é promover uma artrodese numa posição de equilíbrio e conforto na posição sentado. Em geral, a abordagem envolve instrumentação e artrodese via posterior, sem requerer liberação anterior, pela flexibilidade e hipotonia desses pacientes ${ }^{3}$.

A maioria das séries mostra bons resultados com instrumental de Harrington ou Luque, com correção coronal entre 33 e $50 \%{ }^{12-14}$, e espera-se que o uso de instrumentos de $3^{a}$ geração possam beneficiar ainda mais os pacientes. Não foram encontrados registros de séries com uso exclusivo de parafusos pediculares em pacientes com AME. Desde o advento do instrumental de Harrington ${ }^{15}$, muitos outros sistemas de correção foram desenvolvidos. Os parafusos pediculares permitem ao cirurgião uma melhor correção tridimensional da escoliose. Aqueles que defendem seu uso referem como vantagem a fixação das três colunas com melhora da correção coronal sagital e rotacional, menores índices de pseudartrose, menos falha do implante e menos necessidade de órteses no pós-operatório ${ }^{16,17}$.

Pode-se evitar a via anterior, o que já foi descrito em correção de curvas acima de $100^{\circ}$, e superar o fenômeno do crankshaft, que também foi demonstrado por Suk et al. ${ }^{18}$. Evitar a invasão da caixa torácica é benéfico e maximiza a função pulmonar dos pacientes com escoliose, em especial com a derrotação da caixa torácica que pode ser visualizada por tomografia computadorizada ${ }^{19}$, o que é uma grande vantagem para os pacientes com AME pela restrição pulmonar. Outra vantagem do parafuso é estar fora do canal em relação aos fios e ganchos. Além disso, facetas, lâmina e transversa livres de implante permitem maior superfície para decorticação e enxertia sem desestabilizar a montagem ${ }^{19}$.

Embora as taxas de complicação sejam altas no paciente neuromuscular $(24-75 \%)^{10}$, há uma melhora na qualidade de vida dos pacientes ${ }^{20}$. Bridwell et al., em seu trabalho com escoliose neuromuscular flácida progressiva (incluídos nesse grupo AME e distrofia muscular de Duchene), observaram que a maioria dos pacientes e familiares 
acreditam que a cirurgia da coluna melhorou a função, a cosmética, o equilíbrio para sentar e a qualidade de vida ${ }^{13}$.

A literatura mostra que, a longo prazo, ocorre uma perda dos resultados com piora do ângulo de Cobb, e nas séries misturam pacientes com diferentes patologias, o que dificulta seu entendimento específico e criação de escalas próprias para avaliação dos resultados ${ }^{3}$. Para pacientes com AME, um maior seguimento e séries com mais pacientes podem levar a uma melhor compreensão da progressão da curva e do momento para indicar a cirurgia, resultando na obtenção do máximo de correção. Os parafusos pediculares com certeza devem fazer parte do arsenal terapêutico para esses pacientes.

\section{CONCLUSÕES}

O tratamento cirúrgico de pacientes com AME com artrodese via posterior utilizando parafusos pediculares tem grande potencial de correção da deformidade coronal e da obliquidade pélvica. Os resultados do presente estudo mostraram-se superiores aos valores apresentados na literatura com outros instrumentais, e não houve registro de grandes complicações no pós-operatório precoce. Entretanto, há necessidade de melhor avaliação das complicações tardias, perda de correção e melhora do balanço sagital. Tais dados também devem apresentar melhores resultados que os outros métodos de correção.

\section{REFERÊNCIAS}

1. Melki J, Abdelhak S, Shet P. Gene for proximal spinal muscular atrophies maps to chromosome $5 \mathrm{q}$. Nature. 1990;344(6268):767-8.

2. Werdnig G. In: Ashwal S, editor. The founders of child neurology. San Francisco: Jeremy Norman: Child Neurology Society; 1990. p. 383-8.

3. Sucato DJ. Spine deformity in spinal muscular atrophy. J Bone Joint Surg Am. 2007;89 Suppl 1:148-54.

4. Bradford DS, Lonstein JE, Winter RB, Ogilve JW. Moe's the text book of scoliosis and other deformities, 3rd ed. Philadelphia: WB Saunders; 1995.

5. Byers RK, Banker BQ. Infantile muscular atrophy. Arch Neurol. 1961;5:140-64.

6. Weinstein SL. The pediatric spine: principles and practice. 2nd ed. New York: Lippincott Williams \& Wilkins; 2001.

7. Ioos C, Leclair-Richard D, Mrad S, Barois A, Estournet-Mathiaud B. Respiratory capacity course in patients with infantile spinal muscular atrophy. Chest. 2004;126(3):831-7. Comment in: Chest. 2005;127(4):1463; author reply $1463-4$.

8. Gupta MC, Wijesekera S, Sossan A, Martin L, Vogel LC, Boakes JL, et al. Reability of Radigraphic parameters in neuromuscular scoliosis. Spine. 2007;32(6):691-5.

9. Blanke KM, Kuklo TR, Lenke LG, O'Brien MF, Polly Jr DW, Richards BS, et al. Radiographic measurement manual. Spinal Deformity Study Group. USA; 2005. p. 68-9.
10. Mohamad F, Parent S, Pawelek J, Marks M, Bastrom T, Faro F, et al. Perioperative complications after surgical correction in neuromuscular scoliosis. J Pediatr Orhtop. 2007;27(4):392-7.

11.Kim YJ, Lenke LG, Bridwell KH, Riew KD, O'Brien M, et al. CT scan accuracy of "free hand" thoracic pedicle screw placement in pediatric spinal deformity. In: Scoliosis Research Society Annual Meeting; September 2001; Cleveland, Ohio; 2001.

12.Aprin H, Bowen JR, MacEwen GD, Hall JE. Spine fusion in patients with spinal muscular atrophy. J Bone Joint Surg Am. 1982;64(8):1179-87.

13.Bridwell $\mathrm{KH}$, Baldus $\mathrm{C}$, Iffrig TM, Lenke LG, Blanke KRN. Process measures and patient/parent evaluation of surgical management of spinal deformities in patients with progressive flaccid neuromuscular scoliosis (duchenne's muscular dystrophy and spinal muscular atrophy). Spine. 1999;24(13):1300.

14.Bentley G, Haddad F, Bull TM, Seingry D. The treatment of scoliosis in muscular dystrophy using modified Luque and Harrington-Luque instrumentation. J Bone Joint Surg Br. 2001;83(1):22-8.

15.Harrington PR. Treatment of scoliosis: correction and internal fixation by spine instrumentation. J Bone Joint Surg Am. 1962; 44-A;591-610.
16. Hamill CL, Lenke LG, Briedell KH. The use of pedicle screw fixation to improve correction in the lumbar spine of patients with idiopathic scoliosis: is it warranted? Spine. 1996;21(10):1241-9.

17.Gaines RW Jr. The use of pedicle screw internal fixation for operative treatment of spinal disorders. J Bone Joint Surg Am. 2000;82-A(10): 1458-76.

18.Suk EI, Chung ER, Kim JH, et al. Prevention of crankshaft phenomenon with posterior pedicle screw fixation in scoliosis of immature spine: Is it possible? [Presented at the Scoliosis Research Society 37th Annual Meeting, Seattle, WA] 2002. p. 19-21.

19.Lenke LG, Kuklo TR, Ondra S, Polly DW. Rationale behind the current state-of-the-art treatment of scoliosis (in pedicle screw era). Spine. 2008;33(10):1051-4.

20.Mercado E, Alman B, Wright J. Does spine fusion influence quality of life in neuromuscular scoliosis? Spine. 2007;32(19 Suppl)120-5.

\section{Correspondência}

Daniel Cantarelli dos Santos

Avenida Carlos Botelho, 39, Alemães

CEP 13461-40 - Piracicaba (SP), Brasil

E-mail:drdanielcoluna@yahoo.com.br 